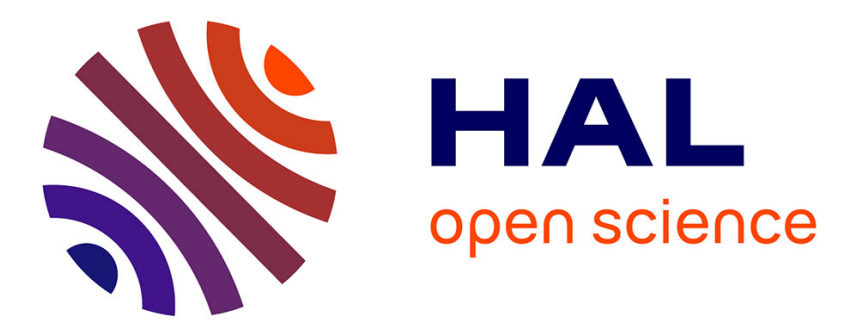

\title{
Multi-time-scale stability analysis and design conditions of a VSC terminal with DC voltage droop control for HVDC networks
}

Yijing Chen, Gilney Damm, Abdelkrim Benchaib, Françoise

Lamnabhi-Lagarrigue

\section{To cite this version:}

Yijing Chen, Gilney Damm, Abdelkrim Benchaib, Françoise Lamnabhi-Lagarrigue. Multi-time-scale stability analysis and design conditions of a VSC terminal with DC voltage droop control for HVDC networks. 53rd IEEE Annual Conference on Decision and Control (CDC 2014), Dec 2014, Los Angeles, United States. pp.3266-3271, 10.1109/CDC.2014.7039894 • hal-01172690

\section{HAL Id: hal-01172690 \\ https://hal.science/hal-01172690}

Submitted on 30 Mar 2021

HAL is a multi-disciplinary open access archive for the deposit and dissemination of scientific research documents, whether they are published or not. The documents may come from teaching and research institutions in France or abroad, or from public or private research centers.
L'archive ouverte pluridisciplinaire HAL, est destinée au dépôt et à la diffusion de documents scientifiques de niveau recherche, publiés ou non, émanant des établissements d'enseignement et de recherche français ou étrangers, des laboratoires publics ou privés. 


\title{
Multi-Time-Scale Stability Analysis and Design Conditions of a VSC Terminal with DC Voltage Droop Control for HVDC Networks
}

\author{
Yijing Chen ${ }^{1}$, Gilney Damm ${ }^{2}$, Abdelkrim Benchaib ${ }^{3}$, Françoise Lamnabhi-Lagarrigue ${ }^{1}$
}

\begin{abstract}
This paper addresses the problem of analyzing the dynamic limitations of a VSC terminal with DC voltage droop control. Thanks to its fast response, DC voltage droop control is usually used to adjust the $\mathrm{DC}$ voltage in case of power imbalance. To ensure the stability of the VSC terminal, the dynamics between the $d q$ currents $\left(i_{l, d q}\right)$ and the DC voltage $\left(u_{c}\right)$ must be well distinguished. Therefore, the choice of the droop control gain $K_{u}$ and the $d q$ current control gains $K_{d q}$ is still a challenging problem. First, we assume that there exist $K_{u}$ and $K_{d q}$ such that a slow and a fast dynamics can be imposed on $u_{c}$ and $i_{l, d q}$, respectively. Then, we propose a novel methodology based on separation of dynamics to prove the existence of these control gains. Subsequently, a detailed stability analysis is carried out via Lyapunov theory, from which a necessary condition and a sufficient condition are derived for the control gains. To verify this methodology, numerical simulations are carried out in MATLAB/SIMULINK.
\end{abstract}

\section{INTRODUCTION}

Voltage-source converter high voltage direct current (VSCHVDC) systems have been greatly promoted in today's electrical networks due to their flexibility, controllability and efficiency. They are specially suitable for long-distance power transmission, interconnection between asynchronous AC networks, underground/undersea cable transmission systems, etc. In order to enlarge power transmission capacity and realize power exchange between multi-suppliers and multi-consumers, the use of multi-terminal HVDC (MTHVDC) systems has been increasingly widely used [1], [2]. However, the control design for MT-HVDC systems is still a major problem. The control scheme should be strong enough to maintain the system in normal operation in case of small-signal perturbations [3] or loss of some terminals [4]. In general, a master controller and local controllers compose the control system where the master one provides information such as the reference values for DC voltage, the active/reactive power to coordinate the operations of the local ones. In an MT-HVDC system, at least one terminal is in charge of regulating the DC-bus voltage level, and hence various control configurations are proposed such as masterslave control scheme, voltage margin method [5].

In this paper, we consider a very popular control technique called DC voltage droop control. It is characterized by the

\footnotetext{
This work is supported by WINPOWER project (ANR-10-SEGI-016).

${ }^{1}$ Y. Chen and F. Lamnabhi-Lagarrigue are with Laboratoire des Signaux et Systèmes (LSS), Supélec, 3 rue Joliot-Curie, 91192 Gif-surYvette, France (tel: +33 1698517 77, e-mail: yijing.chen@1ss.supelec.fr, francoise.lamnabhi-lagarrigue@1ss.supelec.fr).

${ }^{2} \mathrm{G}$. Damm is with Laboratoire IBISC, Université d'Evry-Val d'Essonne, 40 rue du Pelvoux, 91020 Evry, France (e-mail: gilney.damm@ibisc.fr).

${ }^{3}$ A. Benchaib is with ALSTOM GRID/CNAM, France (e-mail: abdelkrim.benchaib@alstom.com).
}

change of power flow in proportion to the change of the DC voltage. The main advantage of DC voltage droop control is that it involves more than one terminal in regulating the DCbus voltage based on their predetermined DC voltage droop characteristics such that more terminals share the duty.

As a matter of fact, the use of DC voltage droop control has been widely studied in the literature [6], [7]. Actually, these control structures belong to the family of vector current control since two loops are usually involved, i.e. an inner current $(i)$ loop and an outer loop. DC voltage droop control considered as an outer loop is used to regulate the DC voltage $\left(u_{c}\right)$ and provide a current reference $i^{*}$ to the current loop. Then, different current loop controllers [8], [9], [10] are proposed to make $i$ converge to $i^{*}$. It is very important to notice that vector current control is derived under the assumption that the current loop has much faster dynamics than the outer one. Thus, the dynamics of $i^{*}$ is neglected when designing the current control scheme. Unfortunately, there is no theoretical demonstration of this assumption. Moreover, it is still unknown which dynamic limitations should be imposed on the current and the DC voltage. This paper is devoted to the stability and dynamic limitation analysis of a VSC station equipped with DC voltage droop control. A sufficient condition for selecting the droop and the current control gains is derived based on singular perturbation theory and Lyapunov theory.

The paper is organized as follows. An averaged statespace model is introduced in Section II. Vector current control scheme with DC voltage droop control is presented in Section III. Section IV gives a detailed stability analysis from which the dynamic limitations on the current and the DC voltage are clarified. We also derive a necessary and a sufficient conditions for choosing the control gains and present the steady-state analysis of the system. The theoretical analysis in this paper is verified by numerical simulations in Section V. Conclusions are drawn in Section VI.

\section{MODELING}

Figure 1 depicts a simplified configuration of a VSC terminal connected to an $\mathrm{AC}$ system where $R_{l}+j L_{l}$ represents the phase reactor and $C$ is the DC capacitor used to reduce the ripple of the DC voltage.

An averaged state-space model established in a synchronous $d q$ reference frame for the VSC terminal is given 




Fig. 1. A VSC terminal connected to AC system.

by [11]

$$
\begin{aligned}
\frac{d i_{l d}}{d t} & =-\frac{R_{l}}{L_{l}} i_{l d}+\omega i_{l q}+\frac{u_{c}}{2 L_{l}} M_{d}-\frac{v_{l d}}{L_{l}} \\
\frac{d i_{l q}}{d t} & =-\frac{R_{l}}{L_{l}} i_{l q}-\omega i_{l d}+\frac{u_{c}}{2 L_{l}} M_{q}-\frac{v_{l q}}{L_{l}} \\
\frac{d u_{c}}{d t} & =-\frac{1}{C}\left[i_{c}+\frac{3}{2} \frac{v_{l d} i_{l d}+v_{l q} i_{l q}}{u_{c}}\right]
\end{aligned}
$$

where $v_{l, d q}, v_{c, d q}$ and $i_{l, d q}$ are the AC system voltages, the converter voltages and the currents through the phase reactor, respectively, $M_{d q}$ are the modulation indices considered as the control inputs and $\omega$ is the frequency of AC system. By convention, $i_{c}$ is positive if the power flows from left to right as shown in Fig. 1.

Remark 1: Due to the physical limitations of the converter itself, the modulation indices should always satisfy $\sqrt{M_{d}^{2}+M_{q}^{2}} \leq 1$ and the domain of operation for $u_{c}$ is restricted to a narrow area, i.e. $u_{c} \in\left[u_{\min }, u_{\max }\right]$.

Remark 2: In the present paper, we want to investigate the local behavior of the converter and hence, for convenience, we consider that the discussed terminal is connected to a controlled current source represented by $i_{c}$. It can be either negative (as a supplier) or positive (as a consumer).

Remark 3: For the sake of simplicity, the $d q$ reference frame is chosen such that $v_{l q}=0$ and $v_{l d}=V_{l, \text { rms }}$. As a consequence, the instantaneous active and reactive powers on the $\mathrm{AC}$ side of the converter are given by

$$
P_{l}=\frac{3}{2} v_{l d} i_{l d} \quad Q_{l}=-\frac{3}{2} v_{l d} i_{l q}
$$

\section{Control Structure}

An inner current loop and an outer loop compose a vector current control system. Various outer loops exist for different control objectives [12], [13]. In this paper, the control goals are to regulate $u_{c}$ in its normal operation region and at the same time, to make $Q_{l}$ follow its reference $Q_{l}^{*}$. Thus, the considered VSC station operates in DC voltage-reactive power mode. By solving (4), the $q$ current reference is obtained as

$$
i_{l q}^{*}=-\frac{2 Q_{l}^{*}}{3 v_{l d}}
$$

As to the $d$ current reference, it is directly given by DC voltage droop control

$$
i_{l d}^{*}=K_{u}\left(u_{c}-u_{c}^{*}\right)
$$

where $K_{u}$ is the droop gain and $u_{c}^{*}$ is the $D C$ voltage reference.
Remark 4: The reference values $Q_{l}^{*}$ and $u_{c}^{*}$ are considered constant in this paper and actually, they are provided by a higher level control called secondary control.

The objective of the current loop is to make $i_{l, d q}$ quickly converge to their varying references $i_{l, d q}^{*}$. Different current controllers have been proposed [8], [9], [10]. In this paper, the conventional current control in [14] is selected. The control signals applied to the converter contain the traditional PI controllers and some compensation terms as

$$
\begin{aligned}
& M_{d}=\frac{2 L_{l}}{u_{c}}\left(v_{d}+\frac{R_{l}}{L_{l}} i_{l d}-\omega i_{l q}+\frac{v_{l d}}{L_{l}}\right) \\
& M_{q}=\frac{2 L_{l}}{u_{c}}\left(v_{q}+\frac{R_{l}}{L_{l}} i_{l q}+\omega i_{l d}+\frac{v_{l q}}{L_{l}}\right)
\end{aligned}
$$

where $v_{d q}$ are

$$
v_{d q}=-K_{p, d q}\left(i_{l, d q}-i_{l, d q}^{*}\right)-K_{i, d q} \int\left(i_{l, d q}-i_{l, d q}^{*}\right)
$$

Finally, the vector current control scheme is built based on (5)-(9).

Remark 5: Note that the dynamics of $i_{l d}^{*}$ is neglected in the current control design since we assume that the currents have much faster dynamics that the DC voltage. Substituting (7)-(9) into the current subsystem leads to

$$
\frac{d i_{l, d q}}{d t}=v_{d q}
$$

where $v_{d q}$ act as additional control inputs to regulate the behaviors of $i_{l, d q}$. It is obvious that the autonomous system (10) is locally exponentially stabilized at $i_{l, d q}^{*}$ if $i_{l, d q}^{*}$ are constant or varying quite slowly. However, as seen in (6), $i_{l d}^{*}$ is given by DC voltage droop control, which means that its dynamics is regulated by $K_{u}$. If $K_{u}$ is quite large, $i_{l d}^{*}$ would have fast dynamics. In this case, the system (10) may become unstable. Therefore, it is necessary to consider the dynamic effect of $i_{l d}^{*}$ on the system stability. In the following section, we will investigate what dynamics $i_{l, d}$ and $i_{l d}^{*}$ should have so that the VSC terminal can operate properly.

\section{STABILITY ANALYSis}

As seen in (6)-(10), the dynamics of $i_{l d}^{*}$ and $i_{l, d q}$ mainly depend on $K_{u}$ and $K_{d q}$. In this section, we investigate the feasible region for these control gains. Before going further, let us study the steady-state condition first.

\section{A. Steady-state condition}

The steady-state values of the system (1)-(3) can be obtained by solving the following algebraic equation

$$
0=-\frac{1}{C}\left[i_{c}+\frac{3}{2} \frac{v_{l d} K_{u}\left(u_{c}-u_{c}^{*}\right)+v_{l q} i_{l q}^{*}}{u_{c}}\right]
$$

which leads to

$$
\begin{aligned}
\bar{u}_{c} & =\frac{3 v_{l d} K_{u} u_{c}^{*}}{3 v_{l d} K_{u}+2 i_{c}}=u_{c}^{*}-\frac{2 u_{c}^{*} i_{c}}{3 v_{l d} K_{u}+2 i_{c}} \\
\bar{i}_{l d} & =-\frac{2 u_{c}^{*} i_{c} K_{u}}{3 v_{l d} K_{u}+2 i_{c}}
\end{aligned}
$$


The equilibrium value of $i_{l q}$, denoted by $\bar{i}_{l q}$, is equal to $i_{l q}^{*}$. Taking the practical feasibility into account, i.e. $\bar{u}_{c} \in$ $\left[u_{\min }, u_{\max }\right], K_{u}$ must satisfy the following condition

\section{Condition 1:}

$$
K_{u}>\operatorname{Max}\left(-\frac{2 i_{c}}{3 v_{l d}}, \frac{2 i_{c} u_{\max }}{3 v_{l d}\left(u_{c}^{*}-u_{\max }\right)}, \frac{2 i_{c} u_{\min }}{3 v_{l d}\left(u_{c}^{*}-u_{\min }\right)}\right)
$$

Remark 6: Notice that Condition 1 is a necessary condition for $K_{u}$. It is obvious that the equilibrium point heavily depends on the choice of the droop gain $K_{u}$ and the DC voltage setpoint $u_{c}^{*}$. As mentioned in the previous section, $u_{c}^{*}$ is provided by the secondary control. In the present paper, $u_{c}^{*}$ is considered as a known constant parameter, which is not used to regulate $\bar{u}_{c}$. Therefore, if a smaller deviation between $\bar{u}_{c}$ and $u_{c}^{*}$ is desired, a larger value for $K_{u}$ should be chosen.

\section{B. Analysis of dynamic limitations and system stability}

In this section, in order to state the main results, two steps are carried out. We first prove that there exist control gains such that the original system (1)-(3) can present a multitime-scale behavior. Subsequently, a sufficient condition for choosing $K_{u}$ and $K_{d q}$ is given to guarantee that the system can be locally stabilized around the equilibrium point in Section IV-A.

Theorem 1: Consider the nonlinear system (1)-(3). For any $K_{u}$ satisfying Condition 1 , there exists $K_{\min }$ such that for any $K_{d q}>K_{\min }$, the proposed control strategy (5)-(9) stabilizes the system around the equilibrium point $\left(\bar{i}_{l d}, \bar{i}_{l q}, \bar{u}_{c}\right)$. Moreover, a multi-time-scale behavior can be found, i.e. a slow and a fast transients.

Proof: To demonstrate this claim, let us substitute the developed control laws (5)-(9) into the actual system (1)-(3). We then get the following closed-loop system

$$
\begin{aligned}
\frac{d i_{l d}}{d t} & =-K_{p d}\left[i_{l d}-K_{u}\left(u_{c}-u_{c}^{*}\right)\right]+\gamma_{d}(\cdot) \\
\frac{d i_{l q}}{d t} & =-K_{p q}\left(i_{l q}-i_{l q}^{*}\right)+\gamma_{q}(\cdot) \\
\frac{d u_{c}}{d t} & =-\frac{1}{C}\left[i_{c}+\frac{3}{2} \frac{v_{l d} i_{l d}+v_{l q} i_{l q}}{u_{c}}\right]
\end{aligned}
$$

where $\gamma_{d q}(\cdot)$ represent the integral parts and for convenience, we set $K_{p d}=K_{p q}=K_{d}$. Let us introduce a new variable $\epsilon$

$$
\epsilon \cdot K_{d}=1
$$

Then, the system (15)-(17) becomes

$$
\begin{aligned}
\epsilon \frac{d i_{l d}}{d t} & =-\left[i_{l d}-K_{u}\left(u_{c}-u_{c}^{*}\right)\right]+\epsilon \gamma_{d}(\cdot) \\
\epsilon \frac{d i_{l q}}{d t} & =-\left(i_{l q}-i_{l q}^{*}\right)+\epsilon \gamma_{q}(\cdot) \\
\frac{d u_{c}}{d t} & =-\frac{1}{C}\left[i_{c}+\frac{3}{2} \frac{v_{l d} i_{l d}+v_{l q} i_{l q}}{u_{c}}\right]
\end{aligned}
$$

When $\epsilon$ is small enough, the derivatives of $i_{l, d q}$ are quite large. By setting $\epsilon=0$ in (19) and (20), $i_{l, d q}$ quickly enter their manifolds $i_{l, d q}^{*}$ and then a reduced model can be deduced from the DC-voltage subsystem as

$$
\frac{d u_{c}^{r}}{d t}=-\frac{1}{C}\left[i_{c}+\frac{3}{2} \frac{v_{l d} K_{u}\left(u_{c}^{r}-u_{c}^{*}\right)+v_{l q} i_{l q}^{*}}{u_{c}^{r}}\right]
$$

Remark 7: The reduced model (22) is locally exponentially stabilized at its equilibrium point $u_{c}^{r}=\bar{u}_{c}$.

Since the reduced model is deduced when $i_{l, d q}$ converge to $i_{l, d q}^{*}$, it is also called a quasi-steady-state model. $i_{l, d q}^{*}$ are the quasi-steady states of $i_{l, d q}$. It is expected that the behavior of the original DC voltage system can be approximated by its reduced model due to its simpler form. Therefore, this requires that $i_{l, d q}$ converge to $i_{l, d q}^{*}$ during the initial interval. In order to analyze the problem more conveniently, let us introduce some new variables:

$$
\begin{aligned}
\tilde{i}_{l, d q} & =i_{l, d q}-i_{l, d q}^{*} \\
\tilde{u}_{c} & =u_{c}-u_{c}^{*}
\end{aligned}
$$

that shift the quasi-steady states of $i_{l, d q}$ to the origin. In these new variables, the full system problem becomes:

$$
\begin{aligned}
\epsilon \frac{d \tilde{i}_{l d}}{d t}= & -\tilde{i}_{l d}+\epsilon K_{u}\left[\frac{3 K_{u}}{2 C} \frac{u_{c}^{*}}{\bar{u}_{c}} \frac{v_{l d}}{\bar{u}_{c}+\tilde{u}_{c}} \tilde{u}_{c}+\frac{3 v_{l d}}{2 C} \frac{\tilde{i}_{l d}}{\bar{u}_{c}+\tilde{u}_{c}}\right] \\
& +\epsilon \gamma_{d} \triangleq f_{d} \\
\epsilon \frac{d \tilde{i}_{l q}}{d t}= & -\tilde{i}_{l q}+\epsilon \gamma_{q} \triangleq f_{q} \\
\frac{d \tilde{u}_{c}}{d t}= & -\frac{3 K_{u}}{2 C} \frac{u_{c}^{*}}{\bar{u}_{c}} \frac{v_{l d}}{\bar{u}_{c}+\tilde{u}_{c}} \tilde{u}_{c}-\frac{3 v_{l d}}{2 C} \frac{\tilde{i}_{l d}}{\bar{u}_{c}+\tilde{u}_{c}} \triangleq f_{u}
\end{aligned}
$$

Now we set

$$
\epsilon \frac{d \tilde{i}_{l, d q}}{d t}=\frac{d \tilde{i}_{l, d q}}{d \tau}
$$

where

$$
\frac{d \tau}{d t}=\frac{1}{\epsilon}
$$

$\tau$ is the introduced new time variable which can be approximated by $\tau=\frac{t}{\epsilon}$. In the $\tau$ time scale, the current subsystem is expressed as

$$
\begin{aligned}
\frac{d \tilde{i}_{l d}}{d \tau}= & -\tilde{i}_{l d}+\epsilon \gamma_{d} \\
& +\epsilon \frac{K_{u}}{C}\left[\frac{3 v_{l d}}{2 u_{c}} K_{u}\left(u_{c}-u_{c}^{*}\right)+i_{c}+\frac{3 v_{l d}}{2 u_{c}} \tilde{i}_{l d}\right] \\
\frac{d \tilde{i}_{l q}}{d \tau}= & -\tilde{i}_{l q}+\epsilon \gamma_{q}
\end{aligned}
$$

The variables $t$ and $\tilde{u}_{c}$ are considered to be varying slowly in the $\tau$ time scale. Setting $\epsilon=0$ restricts $t$ and $u_{c}$ to their initial values, and then the current subsystem is reduced to the following autonomous system

$$
\frac{d \tilde{i}_{l, d q}}{d \tau}=-\tilde{i}_{l, d q}
$$

which is called the boundary-layer model. It is expected that the solutions of (32) can be close to the origin during the boundary-layer interval $\left(t \in\left[0, t_{b}\right]\right)$, and that they can still remain around the origin for the future time $\left(t>t_{b}\right)$ when $t$ and $u_{c}$ slowly vary. This can be achieved since we have

Remark 8: The boundary-layer model (32) is exponentially stable at the origin, uniformly in $\left(t, \tilde{u}_{c}\right)$.

By combining Remarks 7-8 and referring to Theorem 11.4 in [15], there exists $\epsilon_{\min }$ such that for any $\frac{1}{K_{d}}=\epsilon<$ 
$\epsilon_{\min }$, the translated system (25)-(27) is locally asymptotically stable around the origin. It means that the equilibrium of the system (19)-(21), i.e. $\left(\bar{i}_{l d}, \bar{i}_{l q}, \bar{u}_{c}\right)$, is also asymptotically stable. Thus, there exists $K_{\min }=\frac{1}{\epsilon_{\min }}$. This concludes the proof.

Remark 9: From the above proof, $i_{l d}$ exhibits a two-timescale behavior. It starts with a fast transient of $i_{l d}$ moving from the initial value towards $i_{l d}^{*}$. Then, $i_{l d}$ remains close to $i_{l d}^{*}$. Finally, $i_{l d}$ and $i_{l d}^{*}$ converge to their equilibrium $\bar{i}_{l d}$ together. In the simulation section, this two-time-scale behavior will be clearly illustrated.

In the following section, we carry out a further study on the relationship between $K_{u}$ and $K_{d}$. Investigation of feasible regions of $K_{u}$ and $K_{d}$ is made by applying Lyapunov theory. A Lyapunov function is selected as

$$
V=\frac{d}{2} V_{1}+\frac{(1-d)}{2} V_{2}
$$

with $d \in(0,1)$ where $V_{1}$ and $V_{2}$ are derived from the boundary-layer model and the reduced model, respectively.

$$
\begin{aligned}
& V_{1}=\tilde{i}_{l d}^{2}+\tilde{i}_{l q}^{2}+K_{i d} \phi_{d}^{2}+K_{i q} \phi_{q}^{2} \\
& V_{2}=\tilde{u}_{c}^{2}
\end{aligned}
$$

where $\phi_{d q}=\int \tilde{i}_{l, d q}$. Then, the derivative of $V$ can be deduced from (25)-(27)

$$
\dot{V}=-a_{1} \tilde{i}_{l d}^{2}-d \frac{1}{\epsilon} \tilde{i}_{l q}^{2}-a_{2} \tilde{u}_{c}^{2}+a_{3} \tilde{u}_{c} \tilde{i}_{l d}
$$

with

$$
\begin{aligned}
& a_{1}=d\left(\frac{1}{\epsilon}-\frac{3 K_{u}}{2 C} \frac{v_{l d}}{\bar{u}_{c}+\tilde{u}_{c}}\right) \\
& a_{2}=(1-d) \frac{3 K_{u}}{2 C} \frac{u_{c}^{*}}{\bar{u}_{c}} \frac{v_{l d}}{\bar{u}_{c}+\tilde{u}_{c}} \\
& a_{3}=d \frac{3 K_{u}^{2}}{2 C} \frac{u_{c}^{*}}{\bar{u}_{c}} \frac{v_{l d}}{\bar{u}_{c}+\tilde{u}_{c}}-(1-d) \frac{3}{2 C} \frac{v_{l d}}{\bar{u}_{c}+\tilde{u}_{c}}
\end{aligned}
$$

Rewrite $\dot{V}$ as

$$
\dot{V}=-\left(\begin{array}{ll}
\tilde{i}_{l d} & \tilde{u}_{c}
\end{array}\right) P\left(\begin{array}{l}
\tilde{i}_{l d} \\
\tilde{u}_{c}
\end{array}\right)-d \frac{1}{\epsilon} \tilde{i}_{l q}^{2}
$$

where

$$
P=\left(\begin{array}{cc}
a_{1} & -\frac{a_{3}}{2} \\
-\frac{a_{3}}{2} & a_{2}
\end{array}\right)
$$

Lemma 1: If $\epsilon$ is selected such that the matrix $P$ is positive definite, the translated system (25)-(27) asymptotically converges to the origin.

Remark 10: The above lemma is obvious since a positive definite matrix $P$ would result in a negative $\dot{V}$ except at the origin. By applying Barbalat's lemma or LaSalle theorem, it can be proved that the system is asymptotically stable.

In fact, the sufficient condition to be given later is mainly based on Lemma 1. It is known that a symmetric $n \times n$ real matrix $P$ is positive definite if and only if the determinants
TABLE I

THE VSC TERMINAL PARAMETERS.

\begin{tabular}{c|c|c|c|c}
$R_{l}$ & $L_{l}$ & $V_{\text {line }}$ & $C$ & $f$ \\
\hline $9.9 \mathrm{~m} \Omega$ & $3.2 \mathrm{mH}$ & $415 \mathrm{~V}$ & $680 \mu \mathrm{F}$ & $50 \mathrm{~Hz}$
\end{tabular}

TABLE II

BASE QUANTITIES APPLIED IN THE PER-UNIT SYSTEM.

\begin{tabular}{l||l|l|l} 
AC side & $S_{b}=10 \mathrm{kVA}$ & $V_{b}=415 \sqrt{2} \mathrm{~V}$ & $I_{b} \approx 19.67 \mathrm{~A}$ \\
\hline DC side & $S_{d c, b}=10 \mathrm{kVA}$ & $V_{d c, b}=730 \mathrm{~V}$ & $I_{d c, b} \approx 13.70 \mathrm{~A}$
\end{tabular}

of all leading principal minors of $P$ are positive. Thus, in order to get a positive definite $P$, we need

$$
\begin{aligned}
a_{1} & >0 \\
a_{1} a_{2}-\left(\frac{a_{3}}{2}\right)^{2} & >0
\end{aligned}
$$

These two inequalities indicate that if $K_{u}$ and $K_{d}$ satisfy the following condition

Condition 2:

$$
\begin{aligned}
K_{d} & =\frac{1}{\epsilon}>\frac{3 K_{u}}{2 C} \frac{v_{l d}}{\bar{u}_{c}+\tilde{u}_{c}} \\
K_{d} & =\frac{1}{\epsilon}>\frac{a_{3}^{2}}{4 d a_{2}}+\frac{3 K_{u}}{2 C} \frac{v_{l d}}{\bar{u}_{c}+\tilde{u}_{c}}
\end{aligned}
$$

the system (19)-(21) is asymptotically stable at the origin.

Remark 11: It is clear that $\epsilon$ heavily depends on $\tilde{u}_{c}$. If the system is expected to be stable in a larger region, a smaller value should be assigned to $\epsilon$, i.e. a larger value for $K_{d}$. It is also found that a small $\epsilon$ (a large $K_{d}$ ) makes the system have fast responses. However, due to some physical consideration, we cannot improve the responses of the system unlimitedly.

Remark 12: It is worth noticing that the Lyapunov function $V$ is deduced from the reduced model and the boundarylayer model. When $K_{u}$ is fixed, a large value of $K_{d}$ will make $V$ quickly converge to the origin. It also implies that $i_{l, d q}$ enter their manifolds $i_{l, d q}^{*}$ rapidly.

\section{Simulation}

In this section, numerical simulation results are presented to verify the theoretical analysis and Conditions 1-2 derived from the previous section. The parameters of the VSC terminal and the base quantities used in the per-unit system are listed in Table I and Table II, respectively.

For all the simulations, the VSC terminal is supposed to be connected to a controlled current source with $i_{c}=-0.7$ p.u.. The setpoints $u_{c}^{*}$ and $Q_{l}^{*}$ provided by the secondary control are set to 1 p.u. and 0 p.u., respectively. The converter starts from the initial point $\left(i_{l d 0}=0.5\right.$ p.u., $i_{l q 0}=0.1$ p.u., $u_{c 0}=0.95$ p.u.). Taking Condition 1 into consideration, $K_{u}$ must be greater than 0.2075 in order to ensure that the converter acts in its domain of operation, i.e. $u_{c} \in[0.9,1.1]$ p.u.. In the following section, $K_{u}=0.3$ is applied. As seen in the previous analysis, $K_{d}$ can be determined once $K_{u}$ is fixed. Hence, according to Condition 2, a feasible region for $K_{d}$ is given, i.e. $K_{d}>985.7$. In order to demonstrate the 
main results derived from the theoretical analysis, several simulation scenarios are carried out.

Case 1: We choose $K_{d}=23.5$ and $K_{i}=221.6$. Obviously, $K_{d}$ is beyond the given region. $\epsilon$ is then equal to 0.043 .

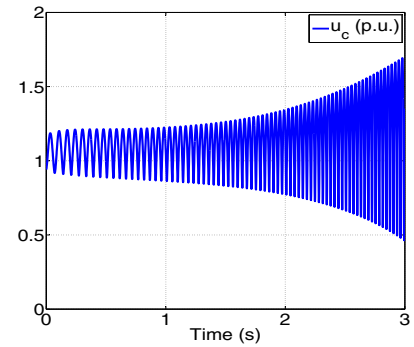

(a) $u_{c}$ response.

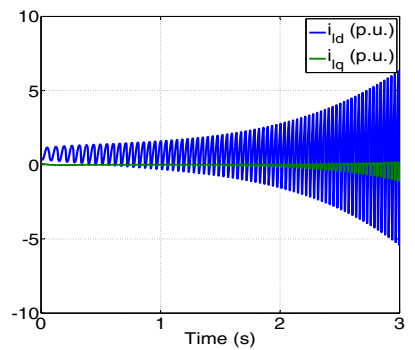

(b) $i_{l, d q}$ response.
Fig. 2. Simulation results with $\epsilon=0.043, K_{d}=23.5$ and $K_{i}=221.6$.

Figures 2(a) and 2(b) illustrate the behaviors of $u_{c}$ and $i_{l, d q}$, respectively. We find that these state variables diverge with time. It is pointed out that in this case, the current controller cannot follow the reference trajectory given by DC voltage droop control. As seen in (25)-(26), the dynamics of $i_{l d}^{*}$ can be considered as a perturbation to its boundary-layer model (32). The divergence of the system variables indicates that the current controller is not strong enough to tolerate this perturbation. It also reveals that not all current controllers can separate the system dynamics.

Case 2: The control gains are selected as $K_{d}=36.2$ and $K_{i}=340.9$. In this case, $K_{d}$ is sill less than 985.7 , and $\epsilon$ is equal to 0.028 .



(a) $u_{c}$ response.

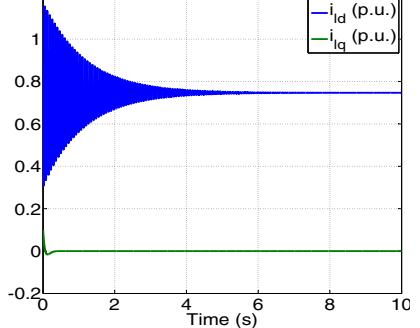

(b) $i_{l, d q}$ response.
Fig. 3. Simulation results with $\epsilon=0.028, K_{d}=36.2$ and $K_{i}=340.9$.

Simulation results are displayed in Figs. 3(a) and 3(b). The trajectories of all the system variables converge to their equilibrium values. It implies that the asymptotic stability of the system can be achieved despite a $K_{d}$ that does not satisfy Condition 2. This is true because our condition is derived from a specific Lyapunov function and that it is not a necessary condition but a sufficient condition. Therefore, it is possible that there exists a $K_{d}$ not satisfying Condition 2 but still ensuring the stability of the system. However, the transient performances of the system variables are not good. Looking at the response of $u_{c}$ in Fig. 3(a), the VSC terminal operates beyond its region of normal operation $([0.9,1.1]$ p.u.) during the interval $t \in(0,1.5) \mathrm{s}$, which may damage electronic devices. On the other hand, the response time of the system is shown to be unsatisfactory.

Case 3: The current control gains are chosen as $K_{d}=986$ and $K_{i}=9.28 \times 10^{3}$ satisfying Condition 2 . In this case, we have $\epsilon=0.001$.

The responses of $i_{l, d}$ and $u_{c}$ are shown in Fig. 4. Let us first focus on the response of $i_{l d}$ in Fig. 4(a). The trajectory of $i_{l d}$ displays a two-time-scale behavior. In contrast to $i_{l d}$ starting from $i_{l d_{0}}=0.5$ p.u., its quasi-steady state $i_{l d}^{*}$ does not start from the same initial value but from $i_{l d_{0}}^{*}=$ $K_{u}\left(u_{c_{0}}-u_{c}^{*}\right)$. Hence, a large discrepancy between $i_{l d_{0}}$ and $i_{l d_{0}}^{*}$ exists at the initial instant, as depicted in Fig. 4(a). It is found that the trajectory of $i_{l d}$ starts with a very fast transient from $i_{l d_{0}}$ to $i_{l d}^{*}$. After this transient, $i_{l d}$ remains close to $i_{l d}^{*}$. For example, the error between $i_{l d}$ and $i_{l d}^{*}$ remains less than 0.114 p.u. after $t=0.005 \mathrm{~s}$. Subsequently, $i_{l d}$ and $i_{l d}^{*}$ converge to the equilibrium value (the red curve) together. As illustrated in Fig. 4(b), the error between $u_{c}$ and $u_{c}^{r}$ remains less than 0.0147 p.u. during the whole simulation. It is pointed out that $u_{c}$ can be well approximated by $u_{c}^{r}$ derived from the reduced model. The response of $i_{l q}$ (not presented

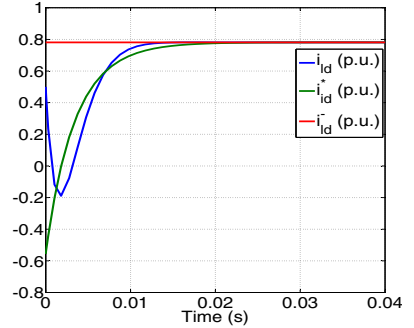

(a) $i_{l d}$ response.

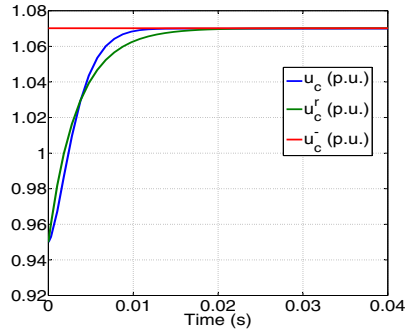

(b) $u_{c}$ response.
Fig. 4. Simulation results with $\epsilon=0.001, K_{i}=9.28 \times 10^{3}$ and $K_{d}=$ 986 corresponding to Condition 2.

here) shows that $i_{l q}$ converges to its reference value, i.e. 0 p.u., after a short transient.

Case 4: As mentioned in Remark 12, $K_{d}$ has a great effect on the convergence speed of the system. In order to evaluate this influence, $K_{d}=1972\left(\epsilon=5 \times 10^{-4}\right)$ and $K_{i}=1.86 \times$ $10^{4}$ are selected by comparison with Case 3 .

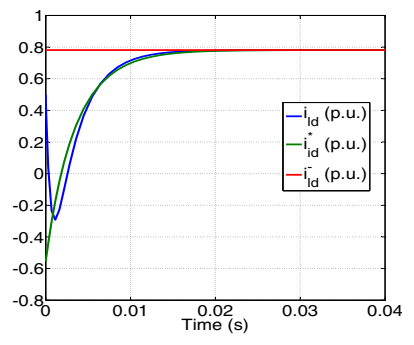

(a) $i_{l d}$ response.

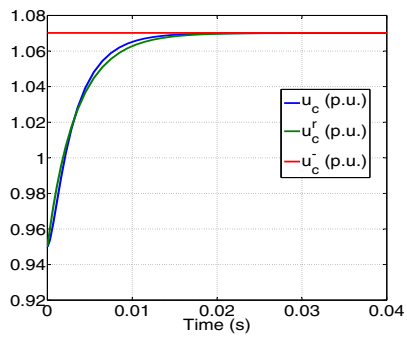

(b) $u_{c}$ response.
Fig. 5. Simulation results with a large $K_{d}=1972, K_{i}=1.86 \times 10^{4}$ and a small $\epsilon=5 \times 10^{-4}$.

The simulation results are shown in Fig. 5. The behavior of $i_{l d}$ in this case is similar to that in Case 3 . The trajectory 
of $i_{l d}$ in Fig. 5(a) also starts with a fast transient from 0.4 p.u. to $i_{l d}^{*}$. After the decay of this transient, $i_{l d}$ always remains around $i_{l d}^{*}$. For $u_{c}$, it remains close to $u_{c}^{r}$.

The main differences between Case 3 and Case 4 are reflected in the following points:

1. As shown in Fig. 6(a), $i_{l d}$ converges to the quasi-steady state $i_{l d}^{*}$ in Case 4 much faster than in Case 3. It is found that after $t=3.1 \mathrm{~ms}$, the error between $i_{l d}$ and $i_{l d}^{*}$ is less than 0.114 p.u., whereas in Case 3, this takes place only after $t=5 \mathrm{~ms}$. It is also clearly shown that $\left|i_{l d}-i_{l d}^{*}\right|$ in Case 4 is smaller than in Case 3 at any instant.

2. The errors between $u_{c}$ and $u_{c}^{r}$ are depicted in Fig. 6(b). A better transient performance is found in Case 4 compared with Case 3 . The discrepancy between $u_{c}$ and $u_{c}^{r}$ in Case 4 is always less than in Case 3 during the whole simulation.

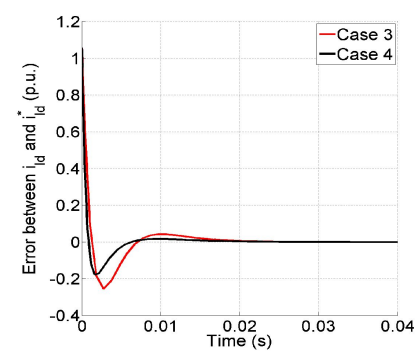

(a) Error between $i_{l d}$ and $i_{l d}^{*}$.

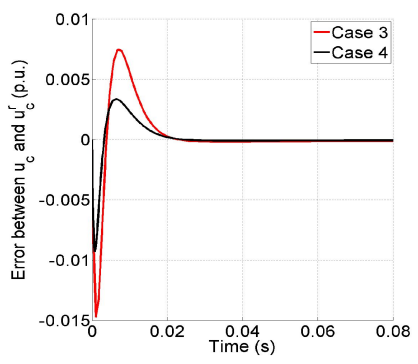

(b) Error between $u_{c}$ and $u_{c}^{r}$.
Fig. 6. Comparison between Case 3 and Case 4 .

By making the comparison between Case 3 and Case 4, we point out that $K_{d}(\epsilon)$ can heavily influence the behaviors of the system. The simulation results show that we can improve the system transient performances by increasing $K_{d}$. This confirms our theoretical analysis since the convergence speed of the system can be accelerated by a large $K_{d}$.

\section{CONCLUSIONS}

In this paper, we consider the problem of regulating the DC-bus voltage with DC voltage droop control. First, we present the conventional vector current control and indicate that the control strategy ensures the stability of the system only when the dynamics between the current and the DC voltage are well distinguished. We then analyze the steady-state condition of the system and find that the current control gain $K_{d}$ has no effect on it. In addition, the droop gain $K_{u}$ and the setpoint $u_{c}^{*}$ determine the steadystate values together. Furthermore, a necessary condition for $K_{u}$ is deduced such that the VSC terminal acts in the region of safe operation. Subsequently, singular perturbation theory is applied to analyze the dynamic limitations of the system. Then, we prove that there exist $K_{u}$ and $K_{d}$ such that the system variables exhibit a multi-time-scale behavior. Finally, we derive a sufficient condition for the control gains by means of Lyapunov theory to guarantee the asymptotic stability of the system. We establish the Lyapunov function based on the boundary-layer model and the reduced model.
It is pointed out that a large $K_{d}$ can improve the system transient performances.

The sufficient condition is verified by carrying out four sets of simulations. It is shown that the derived conditions indeed guarantee the asymptotic stability and make the behaviors of the system well regulated. In order to understand the effects of $K_{d}$ on the dynamics of the system, a comparison is made and the simulation results confirms the theoretical analysis.

In the present paper, the studied VSC is connected to a controlled current source. In the future, it is proposed to consider the VSC terminal in the context of an MT-HVDC system and analyze the effects of the control gains on the problem of stability.

\section{REFERENCES}

[1] W. F. Long, J. Reeve, J. McNichol, R. E. Harrison, D. E. Fletcher, and J. Bowles, "Consideration for implementing multiterminal DC systems," IEEE Transactions on Power Apparatus and Systems, vol. PAS104, pp. 2521-2530, September 1985.

[2] Y. Chen, J. Dai, G. Damm, and F. Lamnabhi-Lagarrigue, "A detailed study on a DC-voltage-based control scheme using a multi-terminal HVDC system for frequency control," in 2013 European Control Conference (ECC), pp. 3530-3535, July 2013.

[3] G. Kalcon, G. Adam, O. Anaya-Lara, S. Lo, and K. Uhlen, "Smallsignal stability analysis of multi-terminal VSC-based DC transmission systems," IEEE Transactions on Power Systems, vol. 27, pp. 18181830, November 2012.

[4] J. Liang, O. Gomis-Bellmunt, J. Ekanayake, and N. Jenkins, "Control of multi-terminal VSC-HVDC transmission for offshore wind power," in 13th European Conference on Power Electronics and Applications, pp. 1-10, 2009.

[5] T. Nakajima and S. Irokawa, "A control system for HVDC transmission by voltage sourced converters," in Power Engineering Society Summer Meeting, vol. 2, pp. 1113-1119, 1999.

[6] T. Haileselassie and K. Uhlen, "Impact of DC line voltage drops on power flow of mtdc using droop control," IEEE Transactions on Power Systems, vol. 27, pp. 1441-1449, August 2012.

[7] E. Prieto-Araujo, F. D. Bianchi, A. Junyent-Ferre, and O. GomisBellmunt, "Methodology for droop control dynamic analysis of multiterminal VSC-HVDC grids for offshore wind farms," IEEE Transactions on Power Delivery, vol. 26, pp. 2476-2485, October 2011.

[8] S. Li, T. Haskew, and L. Xu, "Control of HVDC light system using conventional and direct current vector control approaches," IEEE Transactions on Power Electronics, vol. 25, pp. 3106-3118, December 2010.

[9] Y. Chen, J. Dai, G. Damm, and F. Lamnabhi-Lagarrigue, "Nonlinear control design for a multi-terminal VSC-HVDC system," in 2013 European Control Conference (ECC), pp. 3536-3541, July 2013.

[10] J. Matas, M. Castilla, J. Guerrero, L. Garcia de Vicuna, and J. Miret, "Feedback linearization of direct-drive synchronous wind-turbines via a sliding mode approach," IEEE Transactions on Power Electronics, vol. 23, pp. 1093-1103, May 2008.

[11] V. Blasko and V. Kaura, "A new mathematical model and control of a three-phase AC-DC voltage source converter," IEEE Transactions on Power Electronics, vol. 12, no. 1, pp. 116-123, 1997.

[12] L. Xu, B. Williams, and L. Yao, "Multi-terminal DC transmission systems for connecting large offshore wind farms," in IEEE Power and Energy Society General Meeting - Conversion and Delivery of Electrical Energy in the 21st Century, pp. 1-7, July 2008.

[13] T. M. Haileselassie and M. Molinas, "Multi-terminal VSC-HVDC system for integration of offshore wind farms and green electrification of platforms in the North Sea," in Nordic Workshop on Power and Industrial Electronics (NORPIE), (Espoo, Finland), June 2008.

[14] J. L. Thomas, S. Poullain, and A. Benchaib, "Analysis of a robust DCbus voltage control system for a VSC transmission scheme," in Seventh International Conference on AC and DC Transmission, (London), November 2001.

[15] H. Khalil, Nonlinear Systems. New Jersey: Prentice Hall, 3rd ed., 1996. 